\title{
Os Modelos de Cuidados como Eixo de Estruturação de Atividades Interdisciplinares e Multiprofissionais em Saúde"
}

\author{
Care Models as a Structural Axis for \\ Interdisciplinary and Multi-professional \\ Health Activities
}

Maria Conceição de Oliveiral

PALAVRAS-CHAVE

- Cuidado de saúde;

- Modelos Educacionais;

- Serviços de Integração Docente-Assistencial;

- Educação Médica.

\section{KEY WORDS}

- Delivery of Health Care;

- Models, Educational;

- Teaching Care Integration Services;

- Medical Education.

REVISTA BRASILEIRA DE EDUCAÇ̄̃o MÉDICA
"Trabalho vencedor do Prêmio ABEM/2005 de Educação Médica, no 43ํㅡㄹ Congresso Brasileiro de Educação Médica, realizado no período de 26 a 29 de outubro de 2005, Natal, Rio Grande do Norte, Brasil (2임or).

I Universidade do Planalto Catarinense, Santa Catarina, Brasil. 


\section{INTRODUÇÃO}

A possibilidade de compreender melhor a experiência do adoecer das pessoas é uma das facetas indispensáveis ao que pode ser considerado o maior desafio em saúde no Brasil hoje: um processo de construção/implementação da prática da integralidade em saúde que considere as necessidades das populações.

No Brasil, há um processo de mudança na situação de saúde, com indicadores que mostram a queda da mortalidade infantil e o controle de grande parte das doenças imunopreveníveis. Simultaneamente, há um aumento da expectativa de vida, com as doenças crônicas não transmissíveis (DCNT) ganhando destaque ${ }^{1-4}$.

É inegável a importância de propor ações de saúde adequadas a essa transição demográfica e epidemiológica, e interligadas aos princípios e diretrizes do Sistema Único de Saúde (SUS) e da Estratégia de Saúde da Família (ESF).

A ESF começou a ser implantada em 1994, inicialmente denominada Programa de Saúde da Família. Da forma como é entendida pelo Ministério da Saúde ${ }^{5}$, tem como objetivo geral a reorientação do modelo assistencial de saúde a partir da atenção básica, de acordo com os princípios do SUS, conferindo às Unidades Locais de Saúde uma nova forma de atuação (multiprofissional e interdisciplinar), com definição de responsabilidades dos serviços e da população.

Além disso, uma série de diretrizes curriculares (DC) propõem amplas reformas ${ }^{6}$ no âmbito da formação dos profissionais das áreas de saúde, formação esta mais adequada às necessidades sociais de saúde da população brasileira e convergente com a reorientação do modelo assistencial.

Entre elas, salienta-se a necessidade de construção de projetos pedagógicos que contemplem uma formação interdisciplinar e coerente com a proposta de trabalho multiprofissional de equipes num enfoque complexo da saúde individual e coletiva. Soma-se a isso a necessidade de os cenários de ensino-aprendizagem serem mais diversificados, na tentativa de aprimorar a articulação entre teoria e prática.

Diversos autores têm apontado que a busca de parcerias entre universidades, serviços de saúde e organizações populares é uma das propostas que tem se mostrado como potente estratégia para fortalecer a democracia a partir do estabelecimento de relações horizontalizadas entre os parceiros.

Desse modo, pode-se dizer que há, essencialmente, quatro transições simultâneas no cenário brasileiro, que exigem respostas e reestruturação em vários setores da sociedade:

(1) transição demográfica e epidemiológica da população brasileira;
(2) mudança da estruturação do modelo assistencial do SUS e reorientação do trabalho com a implementação da Estratégia da Saúde da Família e a busca da construção da prática da integralidade em saúde;

(3) mudança do enfoque teórico-metodológico e também prático das questões de saúde na ESF — caráter inter e transdisciplinar na abordagem da saúde com um trabalho multiprofissional em equipe e com participação social/controle social;

(4) mudança da educação dos profissionais de saúde na direção do SUS - Diretrizes para Reforma Curricular nos cursos superiores de saúde; capacitação dos técnicos do SUS; estratégias para ampliar a articulação entre teoria e prática: Integração Docente-Assistencial (IDA); Programa de Articulação Docente-Assistencial (PDA); Projetos UNI; criação de Residências Multiprofissionais em Saúde da Família; proposta de Educação Permanente em Saúde do Ministério da Saúde.

Nesse contexto de mudanças que estão interconectadas, considera-se que um dos temas que merece atenção especial se refere aos caminhos trilhados pelos doentes e suas famílias nos processos de saúde-doença-cuidados.

Esses caminhos - os itinerários terapêuticos ${ }^{7}$ percorridos com vistas à solução dos problemas de saúde - em geral são pouco conhecidos ou relegados a segundo plano, não sendo tema prioritário durante a formação dos profissionais das áreas de saúde e estando pouco presente nas atividades dos técnicos e planejadores dos serviços de saúde. O conhecimento dos cuidados empreendidos é fundamental para que possamos compreender melhor as respostas buscadas pelos clientes usuários do SUS. Em se tratando de doenças crônicas não transmissíveis (DCNT), por exemplo, é imprescindível uma abordagem mais complexa pelos profissionais de saúde em todos os níveis de acompanhamento dos processos individuais e coletivos.

Investigar de modo detalhado as práticas de saúde empreendidas nos processos de saúde/doença na experiência de doentes portadores de DCNT é uma forma de aprofundar o conhecimento que podemos ter sobre os itinerários terapêuticos, sobre os diversos sistemas e/ou modelos de cuidados à saúde utilizados e, também, sobre a vivência experiencial dos doentes e seus familiares.

Uma vez que, na realidade brasileira, costumamos nos referir ao modelo assistencial oficial da saúde como "modelo de atenção", optou-se por denominar esse objeto de "modelos de cuidados", para evitar confusão terminológica. Teixeira ${ }^{8}$ define os modelos de atenção à saúde como "formas de organização das relações entre sujeitos (profissionais de saúde e usuários) mediadas por tecnologias (materiais e não materiais), utilizadas no processo de trabalho em 
saúde, cujo propósito é intervir sobre problemas (danos e riscos) e necessidades sociais de saúde historicamente definidas".

Em termos práticos, a noção de modelos de cuidados aqui utilizada é muito próxima do conceito de modelos de atenção, proposto por Eduardo L. Menéndez"

Cuando desde una perspectiva antropológica hablamos de modelos de atención, nos referimos no solo a las actividades de tipo biomédico, sino a todas aquellas que tienen que ver con la atención de los padecimientos en términos intencionales, es decir que buscan prevenir, dar tratamiento, controlar, aliviar y/o curar un padecimiento determinado, lo cual implica asumir una serie de puntos de partida que contextualizan nuestro análisis de los modelos de atención [...].

Do ponto de vista socioantropológico, os sistemas ou modelos de cuidados devem ser considerados processos dinâmi$\cos$, que possuem graus variados de estruturação. Boff ${ }^{10}$ afirma que não existe vida sem cuidado e o identifica como a essência humana. Ayres ${ }^{11}$, por sua vez, considera fundamental a transformação do cuidado em práticas profissionais, como um importante fator orientador do conjunto de ações de saúde. Não é nosso objetivo aqui empreender uma discussão sobre cuidadores ou teorias do cuidado. Sobre este tema, remetemos aos trabalhos de Cassell ${ }^{12}$, Collière ${ }^{13,14}$ e Karsch $^{15,16}$

Para a discussão do entendimento ampliado sobre os modelos de cuidados, é preciso destacar duas premissas: (1) a possibilidade de articulações teórico-metodológicas para subsidiar mudanças no ensino e nas práticas que contemplem esses modelos; (2) a permanente reflexão (interdisciplinar e multiprofissional) acadêmica, dos serviços e, se possível, das organizações comunitárias sobre o modo como se pensam e estruturam o cuidado e a atenção em saúde.

Este artigo aponta a discussão dessas premissas como uma necessidade a ser suprida, com o objetivo de implementar metodologias de ensino articuladas com o trabalho em saúde (aqui nos referimos aos técnicos de saúde do SUS em todos os níveis da atenção). A segunda proposta é que estas metodologias sejam construídas contextualmente e de modo intersubjetivo pelos sujeitos participantes, que irão enfocar conteúdos que informem práticas em saúde (sem com isso negar, em absoluto, que as próprias práticas modificam pressupostos). Esses conteúdos giram em torno da necessária identificação, compreensão e análise dos modelos de cuidados de saúde empreendidos pela população em geral e pelas pessoas portadoras de DCNT, em particular. Deve ser observado que as doenças do aparelho circulatório representam a principal causa de óbito no Brasil ${ }^{17}$, perfazendo um total de $32 \%$.
Dentre as estratégias consideradas efetivas para implementar as mudanças que podemos denominar "transversais" nos cenários das experiências nacionais na área de saúde, este trabalho se deterá na proposta de integração ensino-serviços local, o Programa Docente-Assistencial Florianópolis (doravante PDA), como locus adequado de discussão, reflexão e pesquisa sobre os modelos de cuidados.

O PDA tem a co-participação da Universidade Federal de Santa Catarina (UFSC) - com os cursos de Medicina, Enfermagem, Nutrição, Farmácia/Análises Clínicas, Psicologia, Serviço Social, Odontologia, e respectivos departamentos do Hospital Universitário da UFSC (HU) e da Secretaria Municipal da Saúde (SMS) — Florianópolis.

Considera-se necessária, portanto, a construção conjunta - interdisciplinar e multiprofissional - de propostas de abordagens teórico-metodológicas de dois grandes temas de discussão, formação, pesquisa e prática de saúde que são confluentes: (1) a temática dos modelos de cuidados de atenção presentes nos itinerários terapêuticos, priorizando as doenças que no Brasil têm forte prevalência de morbi-mortalidade; (2) a temática das mudanças na formação dos profissionais e nas práticas de cuidados e atenção à saúde, a partir do desenvolvimento gradual de novas tecnologias que partem da identificação ampliada de problemas e necessidades de saúde numa perspectiva social e epidemiologicamente orientada para indivíduos e conjuntos sociais portadores dessas doenças.

Em termos mais específicos, iniciou-se a discussão sobre a temática dos modelos de cuidados na Câmara Didático-Pedagógica do PDA a partir de 2005 e optou-se por iniciar a discussão pelo tema "cuidado", visando desencadear esse processo de construção conjunta e solidificação de conteúdos, para uma integração maior entre o ensino e a ótica de novas concepções que contribuam para mudanças cotidianas das práticas de saúde.

O principal resultado que se espera alcançar com esta proposta é que a abordagem dos modelos de cuidados se torne, de fato, um tema transversal na formação acadêmica dos graduandos envolvidos. Isto implica a permanente reflexão sobre: o processo do aprender (apreender) a complexidade da saúdedoença-cuidados; as práticas concretas de saúde de técnicos, alunos e professores que atuam nos serviços; a busca de ações de saúde que construam e fortaleçam o SUS e a ESF.

\section{TEMAS PARA SUBSIDIAR A DISCUSSÃO DOS MODELOS DE CUIDADOS NO PDA — FLORIANÓPOLIS}

\section{Modelos de cuidados e adesão}

Um dos pressupostos que norteiam este trabalho é o de que a população busca diversas soluções práticas para resolver seus problemas de saúde, isto é, há diversos sistemas ou 
modelos de cuidados que são empreendidos. Muitas vezes, durante os processos de adoecer, múltiplas opções de tratamento são utilizadas de forma simultânea, o que gera o que Janzen $^{18}$ denomina um verdadeiro "pluralismo médico", uma "intermedicalidade". Tais opções incluem desde as que se inscrevem na auto-atenção/autocuidados - soluções buscadas no seio das famílias, nos grupos de pertença, na comunidade, no âmbito religioso, com trabalhadores de cura -, até aquelas buscadas nos serviços biomédicos oficiais.

Menéndez ${ }^{9}$ enfatiza que:

En las sociedades lationoamericanas existem diversos modelos de atención de los padecimientos que son considerados en forma aislada y hasta antagónica por el sector salud, en lugar de observar las estrechas relaciones que se dan entre esos modelos a través de las prácticas y representaciones de los sujetos que los utilizan.

Uma das justificativas para a necessidade de construir saberes e práticas que dêem conta destas estreitas relações apontadas por Menéndez é o fato de que nem sempre os tratamentos propostos pelos serviços de biomedicina são efetuados na íntegra pelos pacientes. Quando se trata de doenças crônicas, isto acarreta uma série de dificuldades no manejo adequado dos doentes. Na maior parte das vezes, existe uma lacuna nos serviços de saúde sobre o entendimento do porquê a não adesão está presente no cotidiano das práticas terapêuticas, assim como nos significados que indivíduos, famílias e grupos vivenciam nos processos de saúde, doenças e cuidados.

O episódio de doença deve ser visto como um processo para o qual converge, além de realidades biológicas, psicológicas e de acesso a serviços de saúde, uma série de fatores contextuais, significados socioculturais que circulam no grupo, isto é, aspectos dinâmicos e emergentes em que atores sociais (indivíduo, família, grupo social, profissionais de saúde) e suas ações visam solucionar problemas de saúde. A doença é, portanto, uma experiência significativa, que, para ser apreendida, necessita que se identifiquem e descrevam as formas de cuidados. Como aponta Menéndez ${ }^{9}$, é preciso se debruçar sobre o que "fazem e usam os sujeitos e grupos sociais para atender aos seus padecimentos". Estas práticas (e negociações), muitas vezes, estão ancoradas em atitudes e valores nem sempre plenamente compreendidos pelos profissionais de saúde.

Os modelos explicativos dos processos de saúde e doença que levam em conta os vários tipos de realidades ${ }^{19}$ são essenciais quando tentamos compreender os fatores implicados e as representações presentes nas escolhas terapêuticas dos do- entes em suas práticas de saúde. Além disso, esses processos implicam, em graus variados, mudanças de hábitos na vida das pessoas, com comportamentos, interpretações e escolhas que podem, em muito, dificultar ou impedir a assimilação de novas representações sobre o corpo e sobre o próprio processo de mudanças, o que pode ser um fator de resistência à aquisição de novos hábitos.

A adesão ou não dos pacientes aos tratamentos depende, entre outros fatores, da satisfação obtida junto aos serviços, de questões de vínculo, das respostas às suas necessidades de saúde e, também, do processo de comunicação que ocorre na inter-relação médico-paciente-família e/ou equipe-paciente-família. Além disso, as vivências e respostas encontradas a partir de outras soluções propostas por outros modelos de cuidados que não o biomédico exercem influência sobre a problemática que envolve a adesão.

Por esse prisma confluem as expectativas dos profissionais (desde a sua formação) sobre o modo como os serviços de saúde estão ou devem estar organizados para solucionar problemas específicos e atender às expectativas dos usuários, que, continuamente, avaliam e ressignificam as diferentes respostas por eles buscadas em suas práticas de saúde.

Nesse sentido, estamos tratando de mudanças de "olhares, de saberes e de práticas cotidianas" de trabalho em saúde, estando implicadas estratégias contextualizadas em nível local, além da necessidade da construção de intersubjetividades no cuidado e atenção, pois os doentes e suas famílias são sujeitos (também de suas escolhas terapêuticas). Nessa mesma linha de pensamento, Tesser $\& \mathrm{Luz}^{20}$, ao discutirem os conflitos e dilemas que surgem e que afetam a adesão aos tratamentos, salientam:

[...] tensões e problemas de comunicação e de confiança entre doentes e terapeutas, notoriamente em relação aos médicos. Esses problemas podem ser exemplificados por situações muito comuns: médicos e doentes se desentendem; os processos diagnósticos não raro se frustram, geram situações anômicas e por vezes são iatrogênicos. Feitos os diagnósticos, freqüentemente surgem dilemas terapêuticos, limites terapêuticos ou mesmo ausência de terapêutica. Quando a terapêutica é cientificamente consensual, aparecem de novo problemas quanto a seus riscos, seus custos, suas iatrogenias e suas restrições de abrangência (referenciada na patologia, deixa de lado a "longitudinalidade" do processo saúde-doença do paciente). São comuns os dissensos simbólicos entre profissional e doente e, quando isso não ocorre, aparecem as dificuldades de adesão aos 
tratamentos médicos prolongados, principalmente nas chamadas doenças crônicas, cuja incidência e prevalência parecem só aumentar.

Além da possibilidade de se ampliar o campo epistemológico num eixo que relacione os modelos de cuidados em saúde à construção de práticas de integralidade com ênfase na promoção de saúde, é preciso efetivar pesquisas em saúde nessa área temática, o que implicaria uma pesquisa interdisciplinar de caráter estratégico.

O trabalho interdisciplinar estratégico, que visa subsidiar as políticas públicas de saúde, deverá valorizar, por um lado, as práticas de saúde, os aspectos da subjetividade, os micropoderes e as práticas sociais, como salienta Almeida-Filho ${ }^{21}$, e, por outro, as macrocondições de produção das realidades sociais, as iniqüidades e as desigualdades sociais implicadas ${ }^{22}$.

Pensar a problemática das diferenças e desigualdades socioculturais e de poder no que se refere ao acesso aos serviços de atenção básica de saúde implica, entre outros fatores, levar em conta as práticas cotidianas dos usuários, o entendimento deles sobre as doenças e as escolhas dos modelos de cuidados de saúde, e estes, de acordo com as particularidades de indivíduos e grupos, levarão a certas atitudes.

No caso da DCNT, uma mudança de abordagem que vá além da problemática centrada em indivíduos doentes ou em questões macroestruturais - como mudanças culturais e dificuldades socioeconômicas, inclusive de acesso aos serviços - e que contemple as dinâmicas individuais e populacionais dos processos que resultam em doenças poderá auxiliar na adesão aos tratamentos e em mudanças nos modos de levar a vida, o que pode significar uma grande negociação entre as equipes de saúde e os usuários. Refletir sobre essa negociação é algo que pode (e deve) ser desenvolvido num programa de integração ensino-serviços, como o PDA - Florianópolis.

\section{O CAMPO DA SAÚDE COLETIVA COMO ARTICULAÇÃO INTERDISCIPLINAR ENTRE CIÊNCIAS SOCIAIS E HUMANAS E EPIDEMIOLOGIA}

"[Devemos] procurar relações sistemáticas entre fenômenos diversos, não identidades substantivas entre fenômenos similares."

\section{Clifford GeERTZ}

Quando se quer investigar de que forma os serviços da biomedicina estão organizados e como respondem às necessidades dos usuários em seus itinerários terapêuticos, tendo como premissa a existência de desigualdades sociais no aces- so aos serviços de saúde, levar em consideração uma análise interdisciplinar da saúde não é apenas uma forma de "pensar" a saúde. É uma conduta teórico-metodológica prudente, no sentido formulado por Santos ${ }^{23}$, e particularmente eficaz quando se pensa na consolidação do SUS e das reformas ${ }^{6}$ propostas nas diretrizes curriculares para os cursos de saúde, por exemplo.

A busca de uma adequada avaliação dessas respostas implica, por um lado, visualizar como o ensino na área da saúde e a prática dos profissionais têm abordado o tema dos modelos de cuidados. Por outro, implica a compreensão e integração dos vários saberes dos usuários diante de uma realidade — como no caso da brasileira - em que predomina uma forte desigualdade social. Essa desigualdade talvez possa ser diminuída a partir do fortalecimento da contribuição e articulação entre diferentes abordagens teórico-metodológicas e práticas.

Um campo que precisa se desenvolver de modo mais marcante é aquele que tem sido discutido por Menéndez ${ }^{24}$. Se, por um lado, as práticas sanitárias estão centradas na epidemiologia, mas também consideram as questões macrossociais (econômicas, políticas, educativo-comunicativas e outras), por outro, envolvem simultaneamente o exercício de micropoderes em contextos de desigualdades sociais. Tal fato, como enfatiza o autor, não tem sido contemplado na discussão teóricometodológica da interação epidemiologia/antropologia.

Intencionando preencher essa lacuna, Menénde ${ }^{24}$ propõe uma reformulação do campo de saber epidemiológico em relação ao que denomina uma "epidemiologia sociocultural", principalmente porque as práticas cotidianas dos pacientes se pautam em pluralidades terapêuticas. Nesse sentido, o autor aponta a necessária incorporação, nas rotinas dos sistemas biomédicos, das formas de auto-atenção/autocuidados, uma vez que tal interação existe independentemente da vontade e/ou aceitação do modelo biomédico.

A noção de autocuidado utilizada aqui se refere ao conceito proposto pela OMS (1983) e que é retomado por Kickbusch ${ }^{25}$ :

Se refere às atividades de saúde não organizadas $e$ às decisões sobre a saúde tomadas por indivíduos, famílias, vizinhos, amigos, colegas, companheiros de trabalho, etc.; compreende a automedicação, o autotratamento, o respaldo social na enfermidade, os primeiros auxílios no entorno natural, o que quer dizer, no contexto da vida cotidiana das pessoas.

Ao se buscar uma socioantropologia da saúde crítico-interpretativa ${ }^{26}$, além das várias concepções sobre o corpo, por exemplo, é necessário que as narrativas dos doentes sobre os 
cuidados de saúde sejam conectadas ${ }^{27,28}$ com a vida social, política e individual.

Teixeira ${ }^{8}$ aponta que a identificação de problemas e necessidades de saúde numa perspectiva social e epidemiologicamente orientada pode favorecer a ocorrência de mudanças nas práticas de saúde propriamente ditas, por induzir à ênfase em ações voltadas à intervenção sobre os determinantes dos problemas e não apenas a seus efeitos.

Para Almeida-Filho ${ }^{21,29}$, o "conceito 'modo de vida' (...) poderá resultar em nova vertente da epidemiologia crítica", o que, na visão do autor, significa que a abordagem dos modos de vida implicaria uma reavaliação metodológica por parte da epidemiologia. Esta, então, deveria incorporar outros modelos explicativos que contemplem a complexidade dos processos sociais presentes, o que poderia vir a constituir uma etnoepidemiologia.

Pensar a complexidade exige, evidentemente, a interdisciplinaridade, já que, em muitas situações e contextos específicos, uma série de fatores macrossociais e econômicos pode interferir e ser até mesmo decisiva no que se refere ao bem-estar e às condições de saúde das pessoas.

Menéndez ${ }^{24}$ aponta que a articulação metodológica entre, por exemplo, a antropologia e a epidemiologia exigiria a recomposição dos campos e métodos disciplinares, o que implicaria novas formas de investigação da realidade. Nessa ótica, se produziria bem mais do que uma transferência de métodos entre as disciplinas. O enfoque interdisciplinar ${ }^{30}$ se ajusta como uma poderosa ferramenta para lidar com objetos híbridos de conhecimento e busca alcançar uma explicação mais plausível sobre a complexidade desses objetos.

Quanto à organização da atenção básica, Garnelo e Langdon $^{31}$, nessa mesma linha de pensamento, afirmam que, além do enfoque epidemiológico clássico, há a exigência

\section{[...] da interlocução com temáticas como partici-} pação comunitária e controle social, saberes populares e/ou étnicos sobre saúde e doença, grupos de auto-ajuda e redes sociais, abordagem de patologias crônico-degenerativas, envelhecimento, adolescência, dependência química, violência, relações de gênero, e educação para a saúde, gerando a necessidade de entendimento dos sentidos atribuídos, pelos sujeitos, aos eventos patológicos, às políticas públicas e aos serviços de saúde que lhes são acessíveis.

Pensar a saúde de forma interdisciplinar é fundamental quando se trata de caracterizar, por exemplo, os modelos de autocuidado à saúde que convergem com o atendimento assistencial biomédico.
Simultaneamente, a interdisciplinaridade é fundamental quando se quer investigar, por exemplo, como os serviços da biomedicina estão organizados e de que modo respondem às necessidades dos usuários em seu itinerário terapêutico, tendo como premissa, além da diversidade epidemiológica, a existência de desigualdades sociais no acesso aos serviços de saúde.

\section{O DIÁLOGO E A INTEGRAÇÃO ENTRE O ENSINO E OS SERVIÇOS - A ARTICULAÇÃO TEÓRICO-PRÁTICA COMO MODELO INOVADOR - O EXEMPLO DO PDA - FLORIANÓPOLIS}

Desde a década de 1960, a Organização Mundial da Saúde e a Organização Pan-Americana da Saúde vêm apontando problemas na formação de recursos humanos para a saúde na América Latina e, em particular, na formação de médicos. $\mathrm{O}$ maior problema era uma total falta de planejamento de recursos humanos para a saúde, uma vez que a quantidade de profissionais que se formava dependia dos interesses do sistema educacional e não da necessidade do país ${ }^{32}$.

Sob o predomínio do modelo flexneriano ${ }^{33}$, com o ensino médico centrado no hospital, começou a haver propostas para descentralizar o ensino e a assistência para outros serviços, sendo a formação dos recursos humanos um ponto essencial no desenvolvimento de reformas do setor saúde na América Latina, como descreve Sisson ${ }^{34}$ :

Nos anos 70 aparece na América Latina a estratégia de Integração Docente-Assistencial (IDA) (Vidal e Quiñones, 1986; Rodriguez, 1990; Chaves, 1993; Marsiglia, 1995), para superação das experiências de demonstração dirigidas a pequenos grupos populacionais levadas a cabo pelos departamentos de medicina preventiva, denominadas "laboratórios de comunidade". Esta proposta alternativa visava à inter-relação entre todos os setores da escola médica e parcela significativa dos serviços de saúde, em um contexto regionalizado, com todos os níveis da atenção.

A discussão sobre essa temática se ampliou na América Latina nos anos 1980, e no Brasil começaram a se desenvolver experiências de Integração Docente-Assistencial a partir daí. A ênfase principal era a da relação entre instituições educativas e de serviços como área de formação de conhecimento e de recursos humanos voltados à prática dos serviços de saúde e de ensino como um dos critérios fundamentais no planejamento de mudanças educacionais, visando maior adequação às ne- 
cessidades de saúde da população e buscando romper com o modelo biologicista e superespecializado ${ }^{34,35}$.

A partir do início da década de 1990, houve a implementação de outras estratégias que visavam articular, na área de saúde, as instituições de ensino superior com os serviços. Entre elas, temos um programa político-pedagógico, o Projeto UNI - Uma Nova Iniciativa na Educação dos Profissionais de Saúde -, desenvolvido por 23 instituições de ensino superior na América Latina, das quais seis no Brasil. O UNI "incentivou a construção de modelos inovadores na formação de profissionais de saúde numa parceria da Universidade com os serviços de saúde e com os gestores da sociedade civil organizada nos territórios onde se desenvolvem processos de ensino-aprendizagem" ${ }^{\prime \prime 3}$

Numa linha de propostas situada entre a IDA e a UNI, temos a do Programa de Articulação Docente-Assistencial (PDA), adotado em Florianópolis (SC) inicialmente como modalidade extracurricular e oficializado em maio de 1997, mediante um convênio de Articulação Universidade - Sistema Municipal de Saúde: o PDA — Florianópolis. O que se busca a partir do PDA é uma mudança na formação de profissionais, a fim de que tenham aptidão para trabalhar na promoção da saúde e para resolver problemas na atenção primária. Trata-se de uma parceria para a qual confluem diferentes atores - da área acadêmica, dos serviços de saúde e das organizações comunitárias nas áreas do PDA.

Em 2003, iniciou-se a primeira turma do novo "Currículo Integrado do Curso de Graduação em Medicina", pautado nas novas diretrizes nacionais para os cursos de graduação em saúde. Uma de suas propostas é a inserção precoce do aluno em cenários diversificados de ensino, sendo o Módulo da Interação Comunitária (IC) o eixo integrativo entre os diversos módulos.

Uma dessas diretrizes - extensiva e aplicável aos outros cursos da área da saúde - refere-se justamente à questão de que a integração e a interdisciplinaridade devem ser coerentes com o eixo de desenvolvimento curricular, buscando integrar as dimensões biológicas, psicológicas, políticas, sociais e ambientais. Em outras palavras: que o modelo flexneriano, centrado no mecanicismo, biologicismo, individualismo, especialização e tecnificação, dê lugar à criação de propostas cuja ênfase recaia sobre aspectos de interação, ensino, serviço, comunidade - o que está de acordo com a proposta do PDA.

A ênfase principal recai na reflexão sobre os microprocessos de trabalho, condição necessária para maior articulação entre ensino e serviços. Desse modo, o trabalho aqui proposto e a partir da ação conjunta dos atores envolvidos no Colegiado do PDA - Florianópolis intenciona: (1) diminuir uma lacuna, ao refletir sobre a prática da integralidade como locus da reorientação do modelo assistencial de saúde no Brasil, destacando a necessidade de um planejamento de ações que leve em conta a compreensão dos modelos de cuidados de saúde dos usuários; (2) promover a articulação da perspectiva biomédica com as práticas de auto-atenção dos pacientes.

\section{CONSIDERAÇÕES FINAIS}

Os desafios e mesmo os limites desse trabalho vão desde a definição de matrizes de conhecimento na implementação de reformas curriculares na área de saúde até o processo de reorganização dos serviços de saúde. Este é um processo sinuoso, complexo. Justamente porque pressupõe mudanças no ensino e na forma de organização das práticas de saúde local que levem em conta os modelos de cuidados empreendidos nos itinerários terapêuticos, visando garantir a integralidade do cuidado.

Nesse sentido, pressupõe-se a progressiva construção de um modelo de práticas de assistência em que os pesquisadores na área das ciências sociais e humanas, bem como da saúde coletiva, desempenhem um papel fundamental para a melhoria da formação dos profissionais de saúde e para sugerir propostas de atuação prática dos profissionais, estando implicada nisso a idéia de que se efetuem pesquisas de caráter estratégico.

Este trabalho teve por objetivo discutir a necessidade de desenvolver conhecimento teórico-metodológico e prático que subsidie a identificação, compreensão e análise dos modelos dos cuidados de saúde vivenciados pelos doentes usuários do SUS. Nesse sentido, confluem dois aspectos fundamentais: a presença dessa temática na formação dos estudantes nos cursos de saúde e como essa questão poderá estar colocada no trabalho profissional cotidiano nos vários níveis da assistência do SUS.

A discussão sobre os modelos de cuidados na atenção à saúde (biomedicina e práticas de auto-atenção) e sobre os fatores ligados aos modos de vida dos clientes deverá ser desenvolvida e integrada nos espaços de ensino e de serviços (e talvez de organizações comunitárias), a fim de trazer à luz novos elementos para contribuir na formação dos profissionais, na articulação interdisciplinar entre as ciências sociais e humanas e a saúde coletiva, além de uma adequação maior dos serviços do SUS às realidades encontradas.

A construção de conhecimento interdisciplinar na interface da articulação ensino-serviços que dê conta dos modelos de cuidados de DCNT, por exemplo, tem o caráter de implementação de mudanças de ensino e práticas de saúde numa perspectiva de atenção integral. 
Além disso, a pesquisa interdisciplinar estratégica em saúde é fundamental para a progressiva construção de um modelo em que a saúde coletiva seja abordada de maneira profunda, considerando um amplo número de variáveis e determinantes que fazem dela um objeto de análise extremamente complexo. Os modelos de cuidados podem constituir um dos eixos temáticos para esse tipo de pesquisa.

A proposta a partir daqui é aprimorar essa discussão com um número crescente de atores envolvidos com o ensino e os serviços, como no caso da articulação docente-assistencial promovida pelo PDA. Sua concretização irá refletir o que está destacado como uma das premissas iniciais deste trabalho: "a permanente reflexão (interdisciplinar e multiprofissional) acadêmica e dos serviços sobre o modo como se pensa e se estrutura o cuidado".

Este artigo apresentou proposta aberta, a ser debatida e construída a partir de um tema que permite uma transversalidade de saberes e práticas para o seu enfoque, contribuindo para buscar a construção da integralidade no contexto de consolidação das mudanças convergentes apontadas no início do trabalho. De maneira específica, ele subsidia a discussão que integra as questões macrossociais com as que focalizam as premissas sobre os microprocessos, que constituem as práticas de ensino e serviços cotidianas, com um enfoque centrado no conhecimento sobre a experiência de saúde-doenças-cuidados das pessoas e na reflexão sobre a organização de práticas interdisciplinares e multiprofissionais de saúde pautadas nas reformas curriculares e sanitárias.

\section{REFERÊNCIAS}

1. Brasil. Ministério da Saúde. Secretaria de Vigilância em Saúde. Departamento de Análise de Situação de Saúde. Saúde Brasil 2004: uma análise da situação de saúde. Brasília; 2004.

2. Lessa I org. O adulto brasileiro e as doenças da modernidade: epidemiologia das doenças crônicas não-transmissíveis. São Paulo: Hucitec; 1998.

3. Lessa I. Doenças crônicas não-transmissíveis no Brasil: um desafio para a complexa tarefa da vigilância. Cien Saude Colet 2004; 9(4): 931-43.

4. Rouquayrol MZ, Almeida-Filho N org. Epidemiologia e Saúde. 5. ed. Rio de Janeiro: MEDSI; 1999.

5. Brasil. Ministério da Saúde. Saúde da Família: uma estratégia para a reorientação do modelo assistencial. Brasília: Secretaria da Assistência à Saúde; 1997.

6. Redeunida. Diretrizes Curriculares Nacionais para os Cursos Universitários da Área de Saúde. Londrina; 2002.
7. Augé M. L'Antropologie de la Maladie. In: L'Homme. Número espécial. Antropologie. État de Lieus. Paris: Navarim/Livre de Poche, 1986.

8. Teixeira CF. Modelos de atenção voltados para a qualidade, efetividade, eqüidade e necessidades prioritárias de saúde. Caderno da 11a Conferência Nacional de Saúde, Brasília: Conselho Nacional de Saúde/ Ministério da Saúde; 2000: 261-281.

9. Menéndez E. Modelos de atención de los padecimientos: de exclusiones teoricas y articulaciones prácticas. Cienc Saude Colet 2003; 8(1):185-208.

10. Boff L. Saber cuidar: Ética do humano: compaixão pela terra. Petrópolis: Vozes; 2000.

11. Ayres JRC. Sujeito, intersubjetividade e práticas de saúde. Cad Saude Colet 2001;6(1): 67-72.

12. Cassel EJ The nature of suffering and the goals of medicine. N Engl J Med 1982;306: 639-45.

13. Colière M. Promover a Vida: da prática de mulheres de virtude aos cuidados de enfermagem. Lisboa: LIDEL; 1999.

14. Colière M. Cuidar... a primeira arte da vida. Lisboa: Lusociência; 2003.

15. Karsch U org. Envelhecimento com Dependência: Revelando Cuidadores. São Paulo: EDUC; 1998.

16. Karsch U. Idosos dependentes famílias e cuidadores. Cad. Saúde Pública 2003; 19(3):861-6.

17. Zago MA. A pesquisa clínica no Brasil. Cien Saude Colet 2004; 9(2):363-74.

18. Janzen JM. The Quest of Therapy: Medical Pluralism in Lower Zaire. Berkeley: University of California Press; 1982.

19. Kleinman A. Patients and Healers in the Context of Culture. Berkeley. University of California Press; 1980.

20. Tesser CD, Luz MT. Uma introdução às contribuições da epistemologia contemporânea para a medicina. Cien Saude Colet 2002; 7(2):363-72.

21. Almeida-Filho N. A Ciência da Saúde. São Paulo: Hucitec; 2000.

22. Minayo MCS, Assis SG, Deslandes SF, Souza ER. Possibilidades e dificuldades nas relações entre ciências sociais e epidemiologia. Cien Saude Colet 2003; 8(1):97-108.

23. Santos BS org. Conhecimento prudente para uma vida decente. "Um discurso sobre a ciência” revisitado. São Paulo: Cortez; 2004.

24. Menéndez E. Antropologia médica e epidemiologia. Processo de convergência ou processo de medicalização? In: Alves PC, Rabelo MC. orgs. Antropologia da Saúde. Traçando Identidade e Explorando Fronteiras, Rio de Janeiro: Relume Dumará; 1998 p. 71-94. 
25. Kickbusch I. Promoción de la salud: una antología. Washington, D.C: OPS; 1996.

26. Lock M, Scheper-Hughes N. A Critical-Interpretative Approach in Medical Anthropolofy: Ritual and Routines of Discipline and Dissent. In: Johson TM, Sargent E. org. Medical Anthropology: Contemporary Theory and Method. New York: Praeger Publications; 1990. p. 47-72.

27. Bibeau G. A step toward thick thinking: form web of significance to connections across dimensions. Med Anthropol Q 1988; 2:402-16.

28. Young A. The discourse on stress and the reproduction of conventional knowledge. Soc Sci Med 1980; 148: 133-46.

29. Almeida-Filho N. Modelos de determinação das doenças crônicas não-transmissíveis. Cien Saude Colet 2004; 9(4):865-84.

30. Sevalho Gil, Castiel L. Epidemiologia e antropologia médica: a in(ter)disciplinaridade possível. In: Alves PC, Rabelo MC. orgs. Antropologia da Saúde. Traçando identidade e explorando fronteiras. Rio de Janeiro: Relume Dumará; 1998. p. 47-70.

31. Garnelo L, Langdon EJ. A antropologia e a reformulação de práticas sanitárias na tensão básica; 2003. (Mimeo).

32. Almeida MJ. Educação Médica e saúde: Limites e Possibilidades das propostas de mudança. São Paulo; 1997 Doutorado [Tese] — Universidade de São Paulo.
33. Flexner A. Medical Education in the United States of America and Canada. Carnegie Foundation for the Advancement of Teaching; 1910.

34. Sisson MC. Avaliação da implantação do Programa de Saúde da Família no Programa Docente-Assistencial de Florianópolis. São Paulo; 2002 Doutorado [Tese] — Universidade São Paulo.

35. Marsiglia RG. Relação ensino-serviços: dez anos de integração docente-assistencial IDA no Brasil. São Paulo: Hucitec; 1995.

36. Silva KL, Sena RR. A diversificação de cenários de ensino-aprendizagem como estratégia para articular teoria e prática. Olho Mágico 2004; 11(1/2): 9-16.

Obs.: A autora era vinculada à UFSC à época de realização deste trabalho.

\section{CONFLITOS DE INTERESSE}

Declarou não haver

\section{ENDEREÇO PARA CORRESPONDÊNCIA}

Maria Conceição de Oliveira

Rua Lauro Linhares, 728 - sala 305

Trindade - Florianópolis

CEP. 88063-100 / SC

E-mail: conceliveira@yahoo.com.br 